\title{
GOBERNANZA INDIRECTA DE CRISIS TRANSNACIONALES: LA OPS Y LA OMS FRENTE A LA PANDEMIA DE COVID-19 EN AMÉRICA LATINA
}

\author{
INDIRECT GOVERNANCE OF TRANS-NATIONAL CRISES: \\ THE PAHO AND WHO RESPONSE TO THE COVID-19 \\ PANDEMIC IN LATIN AMERICA
}

\author{
GOUVERNANCE INDIRECTE DE LA CRISE \\ TRANSNATIONALE: L'OPS ET L'OMS FACE À LA \\ PANDÉMIE COVID-19 EN AMÉRIQUE LATINE
}

\author{
LAURa Zamudio GonZÁlez \\ Universidad Iberoamericana \\ laura.zamudio@ibero.mx
}

RESUMEN: Las organizaciones intergubernamentales, regionales e internacionales desempeñan un papel activo en la gobernanza de las crisis transnacionales. En el contexto de la pandemia de Covid-19 en América Latina, la Organización Mundial de la Salud (oms) y la Organización Panamericana de la Salud (ops) se han vinculado con múltiples actores y niveles de decisión, materializando lo que la literatura de gobernanza global identifica como gobernanza indirecta por orquestación. El artículo muestra que, en la práctica, los mecanismos de orquestación han construido esquemas de coordinación y acción heterogéneas que, en situaciones de crisis transnacional, permiten a estas organizaciones sumar recursos, capacidades y autoridad.

Palabras clave: gobernanza indirecta; orquestación; crisis transnacionales; organizaciones internacionales; autoridad; Covid-19; América Latina.

AвSTRACT: Inter-governmental, regional and international organizations play an active role in the governance of transnational crises. In the context of the Covid-19 pandemic in Latin America, the World Health Organization (wHo) and the Pan-American Health Organization (PAHO) have been linked with multiple actors and levels of decision-making, putting into practice what the literature on global governance refers to as indirect governance by orchestra- 
tion. The article shows that, in practice, the mechanisms of orchestration have established heterogeneous models of coordination and action that, in situations of transnational crisis, allow these organizations to bring together resources, capacities and authority.

Keywords: indirect governance; orchestration; transnational crises; international organizations; authority; Covid-19; Latin America.

\section{Traducción de Fionn Petch, CM Idiomas}

Résumé: Les organisations intergouvernementales, régionales et internationales jouent un rôle actif dans la gouvernance des crises transnationales. Dans le contexte de la pandémie de Covid-19 en Amérique latine, l'Organisation mondiale de la santé (OMS) et l'Organisation panaméricaine de la santé (OPS) ont été liées à de multiples acteurs et niveaux de décision, matérialisant ce que la littérature sur la gouvernance mondiale identifie comme une gouvernance indirecte par orchestration. L'article montre que, dans la pratique, les mécanismes d'orchestration ont construit des schémas de coordination et d'action hétérogènes qui, dans des situations de crise transnationale, permettent à ces organisations de mettre en commun des ressources, des capacités et de l'autorité.

Mots clefs: gouvernance indirecte ; orchestration ; crises transnationales ; organisations internationales ; autorité ; Covid19 ; Amérique latine.

Traducción de Rafael Segovia, CM Idiomas

Fecha de recepción: noviembre de 2020

Fecha de aceptación: febrero de 2021 
$\mathrm{L}$ a crisis sanitaria global ocasionada por la Covid-19 ha demostrado que la gobernanza de los problemas transnacionales ${ }^{1}$ requiere de esfuerzos colectivos y acciones coordinadas entre diversos gobernadores, entendidos como "autoridades que ejercen poder a través de fronteras, con el propósito de afectar las políticas ". ${ }^{2}$ La lectura tradicional que considera los Estados como únicos gobernadores, descuida el hecho de que estamos observando una arena política internacional compuesta de organizaciones, empresas transnacionales, asociaciones profesionales, grupos de defensoría, etcétera, que también son agentes activos de la gobernanza transnacional, pues toman decisiones, establecen reglas y diseñan esquemas de coordinación entre ellos y con los Estados para dar atención a problemas.

La gobernanza transnacional no es entonces, simplemente, la "suma de organizaciones, instrumentos de política, mecanismos financieros, reglas, procedimientos y normas", ${ }^{3}$ sino el resultado de la interacción e interdependencia alcanzada entre agentes diversos, inmersos en determinadas lógicas de coordinación y cooperación. ${ }^{4}$

La gobernanza de las crisis transnacionales ofrece un campo de estudio interesante para observar algunas dinámicas de interacción y coordinación entre diferentes tipos de

${ }^{1}$ Usamos los conceptos de "crisis transnacional" y "gobernanza transnacional” en sintonía con el vocabulario empleado por las y los especialistas que alimentan el debate en discusión en este artículo. Sin embargo, su definición es similar a la de "crisis global" y "gobernanza global", preferidos en otras bibliografías en el campo de las relaciones internacionales.

${ }^{2}$ Deborah D. Avant, Martha Finnemore y Susan K. Sell, Who Governs the Globe? Cambridge, University Press, 2010.

${ }^{3}$ Adil Najam, Mihaela Papa y Nadaa Taiyab, Global Environmental Governance: A Reform Agenda, Winnipeg, International Institute for Sustainable Development (IISD), 1999.

${ }^{4}$ Deborah D. Avant, Martha Finnemore y Susan K. Sell, op. cit.; James N. Rosenau y Ernst-Otto Czempiel (eds.), Governance without Government: Order and Change in World Politics, Cambridge, University Press, 1992; Thomas Weiss, Global governance. In What? Why? Whither?, Cambridge, Polity Press, 2013. 
gobernadores, más allá de los Estados y demás agentes. Las y los teóricos de las organizaciones ${ }^{5}$ y de la acción colectiva ${ }^{6}$ han demostrado que la coordinación es clave para explicar el éxito y el fracaso en los esfuerzos de cooperación. Sin embargo, ni la bibliografía sobre la gestión de crisis transnacionales ${ }^{7}$ ni la de Relaciones Internacionales sobre cooperación ${ }^{8}$ han analizado con amplitud la manera en que diversos go-

${ }^{5}$ Arjen Boin y Fredrik Bynander, "Explaining Success and Failure in Crisis Coordination", Geografiska Annaler, vol. 97, núm. 1, 2014, pp. 123135; Tom Christensen, Per Laegreid y Lisa H. Rykkja, "Organizing for Crisis Management: Building Governance Capacity and Legitimacy", Public Administration Review, vol. 76, núm. 6, 2016, pp. 887-897; Per Laegreid, et al., Organizing for Coordination in the Public Sector: Practices and Lessons from 12 European Countries, Basingstoke, Palgrave Macmillan, 2014.

${ }^{6}$ Mancur Olson, The Logic of Collective Action: Public Goods and the Theory of Groups, Cambridge, Harvard University Press, 1965; Todd Sandler, Global Collective Action, Cambridge, University Press, 2004.

${ }^{7}$ Arjen Boin, "The New World of Crises and Crisis Management: Implications for Policymaking and Research", Review of Policy Research, vol. 26, núm. 4, 2009, pp. 367-377; Arjen Boin y Fredrik Bynander, op. cit.; Arjen Boin, et al., The Politics of Crisis Management: Public Leadership under Pressure, Cambridge, University Press, 2016; Daniel Nohrstedt, et al., "Managing Crisis Collaboratively: Prospect and Problems-A Systematic Literature Review", Perspectives on Public Management and Governance, vol. 1, núm. 4, 2018, pp. 257-71; Uriel Rosenthal, Arjen Boin y Louise K. Comfort (eds.), Managing Crisis: Threats, Dilemmas, Opportunities, Springfield, Charles C. Thomas Publishers, 2001; Uriel Rosenthal, Paul 't Hart y Michael T. Charles, "The World of Crises and Crisis Management", en Uriel Rosenthal, Michael T. Charles y Paul 't Hart (eds.), Coping with Crises: The Management of Disasters, Riots and Terrorism, Springfield, Charles C. Thomas Publishers, 1989, pp. 3-36.

${ }^{8}$ Robert O. Keohane, After Hegemony. Cooperation and Discord in the World Political Economy, Princeton, University Press, 1984; Stephen D. Krasner (eds.), International Regimes, Ithaca, Cornell University Press, 1983; Robert Axelrod, The Evolution of Cooperation, Nueva York, Basic Books, 1983; Kenneth A. Oye, Cooperation under Anarchy, Princeton, University Press, 1986; Robert Jervis, "Cooperation under the Security Dilemma", World Politics, vol. 30, núm. 2, 1978, pp. 167-214; Kenneth A. Oye, "Explaining Cooperation under Anarchy: Hypotheses and Strategies", World Politics, vol. 38, núm. 1, 1985, pp. 1-24; Oran, R. Young, International Cooperation, Ithaca, Cornell University Press, 1989. 
bernadores globales se dividen tareas, delegan, coordinan, compiten y cooperan para hacer frente a situaciones cargadas de incertidumbre y fragmentación. ${ }^{9}$ El estudio de las modalidades de gobernanza, específicamente, la gobernanza indirecta por orquestación, ${ }^{10}$ parece ofrecer una respuesta al problema de coordinación en este tipo de escenarios.

El objetivo de este artículo es contribuir al estudio de la gobernanza transnacional, mediante el análisis de un caso de vinculación y coordinación organizacional para el manejo de crisis, específicamente, el papel de la Organización Mundial de la Salud (oms) y la Organización Panamericana de la Salud (ops), su oficina regional, ante la pandemia ocasionada por la Covid-19 en América Latina y el Caribe.

Con base en referencias sobre gobernanza transnacional, se busca demostrar que, en la gobernanza de la pandemia, tanto la oms como la ops actuaron como gobernadores y, al vincularse con otros actores, materializaron una forma de gobernanza indirecta conocida como orquestación.

El artículo se centra en el análisis de las funciones de gestión de crisis, conferidas a la oms y a la ops por el Reglamento

${ }^{9}$ Eva Karin Olsson y Bertjan Verbeek, "International Organizations and Crisis Management", en Bob Reinalda (ed.), Routledge Handbook of International Organization, Nueva York, Routledge, 2013, pp. 324-336; Eva Karin Olsson, "Transboundary Crisis Networks: The Challenge of Coordination in the Face of Global Threats", Risk Management, vol. 17, núm. 2, 2015, pp. 91-108.

${ }^{10}$ Kenneth W. Abbott y Duncan Snidal, "The Governance Triangle: Regulatory Standards Institutions and the Shadow of the State", en Walter Mattli y Ngaire Woods (eds.), The Politics of Global Regulation, Princeton, University Press, 2009, pp. 44-88; Kenneth W. Abbott y Duncan Snidal, "International Regulation without International Government: Improving IO Performance through Orchestration", Review of International Organizations, vol. 5, núm. 3, 2010, pp. 315-344; Kenneth Abbott, et al., "Orchestration: Global Governance through Intermediaries", 2012, http://ssrn.com/ab stract=2125452 (consulta del 20 de febrero de 2021); Kenneth W. Abbott, et al., International Organizations as Orchestrators, Cambridge, University Press, 2015; Kenneth W. Abbott, et al., "Two Logics of Indirect Governance: Delegation and Orchestration”, British Journal of Political Science, vol. 46, núm. 4, 2016, pp. 719-729. 
Sanitario Internacional (RSI) ${ }^{11}$ y por el Comité Permanente entre Organismos (IASC por sus siglas en inglés), ${ }^{12}$ específicamente, las tareas de investigación y monitoreo de los brotes epidémicos. La idea entonces es comprender y observar, en este caso, los mecanismos de participación de la oms y la ops en estructuras de coordinación. Como consecuencia, no estudiamos los resultados de sus acciones sobre las decisiones tomadas por los gobiernos nacionales.

La pregunta que guía el artículo es: ¿qué mecanismos de coordinación orquestados por las oms y la ops fomentaron esquemas de gobernanza indirecta en el contexto de la pandemia, entendida como crisis transnacional, y qué fortalezas y retos se identifican? Se propone responder a esta pregunta de manera exploratoria, dado que es un estudio de caso, al sistematizar los mecanismos que la oms y la ops generaron para coordinar la lucha contra la Covid-19 en América Latina y el Caribe.

Así, el caso contribuye al debate sobre las formas y mecanismos de gobernanza transnacional, específicamente la gobernanza indirecta desde organizaciones intergubernamentales, en contextos de crisis, y aporta también a la literatura sobre la autonomía y agencia ${ }^{13}$ de dichas organizaciones, ${ }^{14}$

${ }^{11}$ Organización Mundial de la Salud, oms, Reglamento Sanitario Internacional, Documento WHA 58.3, Ginebra, 2005.

${ }^{12}$ El IAsc es lo más cercano a una estructura de gobierno y está integrada por 18 instancias diferentes que discuten estrategias, objetivos y propuestas de acción. Creado en 1991 por la Resolución 46/182 de la Asamblea General de las Naciones Unidas, es un foro de coordinación en cuestiones humanitarias. Las instancias que toman decisiones en este nivel son: siete agencias de la onU, el Comité y la Federación Internacional de la Cruz Roja, el Banco Mundial, el Representante del Secretario General (SG) de la ONU para Derechos Humanos y organizaciones internacionales no gubernamentales.

${ }^{13}$ En este caso, agencia se refiere a la capacidad de decisión y acción de las organizaciones.

${ }^{14}$ Michael Barnett y Martha Finnemore, "The Politics, Power and Pathologies of International Organizations", International Organization, vol. 53, núm. 4, 1999, pp. 699-732; Michael Barnett y Martha Finnemore, 
en tanto se conciben como gobernadores de la gobernanza, o como actores con autoridad frente a los Estados, en contraste con el paradigma teórico dominante en la disciplina. ${ }^{15}$

El artículo se divide de la manera que a continuación se explica. En la primera sección se presenta el debate de las Relaciones Internacionales (RI) sobre el papel de las organizaciones internacionales (OI) como gobernadores, así como las modalidades analíticas de delegación y orquestación. Posteriormente, se identifican algunos mecanismos de coordinación aplicados por la oms y la ops en el manejo de la pandemia en América Latina y el Caribe, lo que permitirá señalar fortalezas y retos de la orquestación como modalidad de gobernanza indirecta.

\section{GOBERNANZA TRANSNACIONAL, GOBERNANZA INDIRECTA Y OI}

Las oI participan en la gobernanza transnacional como gobernadores debido a que son instancias autónomas de autoridad. ${ }^{16}$ Tal como lo sostienen Barnett y Finnemore, "las burocracias materializan una forma de autoridad, la autoridad racional-legal, que la modernidad ve como particularmente legítima y buena". ${ }^{17}$ Se trata de una forma de autoridad que descansa en

Rules for the World: International Organizations in Global Politics, Ithaca, Cornell University Press, 2004; Jarle Trondal, "The Autonomy of Bureaucratic Organizations: An Organization Theory Argument", Journal of International Organizations, vol. 5, núm. 2, 2014, pp. 54-68; Joel Oestreich (ed.), International Organizations as Self Directed Actors. A framework for Analysis, Nueva York, Routledge, 2012.

${ }^{15}$ John J. Mearsheimer, "The False Promise of International Institutions", International Security, vol. 19, núm. 3, 1994-1995, pp. 5-49; Robert O. Keohane, op. cit.; Robert O. Keohane, "Reciprocity in International Relations”, International Organization, vol. 40, núm. 1, 1986, pp. 1-27.

16 Michael Barnett y Martha Finnemore, "The Politics, Power, and Pathologies of International Organizations”, art. cit., p. 10.

${ }^{17}$ Loc. cit. 
procedimientos legales y reglas impersonales, en torno a las cuales las burocracias crean conocimiento social. ${ }^{18}$

El control burocrático sobre la información y la experiencia es también una fuente de autonomía y poder de las oI, ya que mientras "el conocimiento técnico experto ayuda a las burocracias a cumplir más eficientemente con las directivas de los políticos, Weber estableció que también daba a las burocracias poder sobre los políticos (y otros actores) y habilitaba a las burocracias a forjar políticas, y no solamente a implementarlas". ${ }^{19}$

Con base en estos atributos, las burocracias ejercen tres tipos de poder: 1) clasifican el mundo, creando categorías de actores y de acción; 2) fijan significados en el mundo social y 3) articulan y difunden normas, principios y actores. ${ }^{20}$

Así, el papel de estas agencias especializadas y su capacidad para estructurar conocimiento social relevante da sustento a los llamados a favor del "multilateralismo científico", 21 y del "multilateralismo efectivo". ${ }^{22}$ Si bien no son la panacea y, como sostienen las y los teóricos de los complejos de regímenes, ${ }^{23}$ la presencia de actores burocráticos aumenta también la densidad institucional y puede actuar tanto a fa-

${ }^{18}$ Loc. cit.

${ }^{19}$ Ibid., p. 11.

${ }^{20}$ Ibid., p. 13.

${ }^{21}$ Gilberto Rodrigues y Alberto Kleiman, "Covid-19: ¿una nueva oportunidad para el multilateralismo?", Foreign Affairs Latinoamerica, vol. 20, núm. 3, 2020, pp. 36-46.

${ }^{22}$ Joachim A. Koops, "Inter-organizational Approaches", en Knud E. Jørgensen y Katie V. Laatikainen (eds.), The Routledge Handbook of Europe and Multilateral Institutions, Londres, Routledge, 2013, pp. 71-85; Rafael Biermann, "Designing Cooperation among International Organizations. Autonomy Concerns, the Dual Consensus Rule, and Cooperation Failure", Journal of International Organizations Studies, vol. 6, núm. 2, 2015, pp. 45-66.

${ }^{23}$ Kal Raustiala y David G. Victor, "The Regime Complex for Plant Genetic Resource's", International Organization, vol. 58, núm. 2, 2004, pp. 277-309; Sebastian Oberthür y Olav Schram Stokke (eds.), Managing Institutional Complexity: Regime Interplay and Global Environmental Change, Cambridge, MIT Press, 2011. 
vor de la complementariedad, como en su contra, con problemas de duplicidad, superposición y competencia entre los agentes. Es decir, la interacción de los actores burocráticos es compleja.

En la literatura sobre la gobernanza transnacional, la interacción entre los agentes es clave también para entender los resultados que pueden alcanzar. ${ }^{24}$ Más allá del tipo de agente, o sus capacidades individuales, la vinculación entre ellos posibilita nuevas dinámicas de acción, con efectos en resultados. ${ }^{25}$ De allí que, para los propósitos de esta investigación, resulte relevante comprender dos modalidades analíticas de gobernanza indirecta: delegación y orquestación. ${ }^{26}$ Como se verá enseguida, la orquestación implica la vinculación de actores sobre una base voluntaria, donde el orquestador carece de controles legales sobre ellos.

La gobernanza indirecta por delegación se efectúa mediante la existencia de un contrato formal, a través del cual un gobernador (o principal) concede autoridad de manera condicionada a un tercero (o agente), y lo empodera para actuar en su nombre. ${ }^{27} \mathrm{El}$ principal puede rescindir el contrato cuando el agente se aparta de los objetivos trazados por él. Se trata de una modalidad fundamentalmente jerárquica donde, al menos formalmente, el principal controla al agente, si bien son los agentes los que proveen los incentivos para

${ }^{24}$ Deborah D. Avant, Martha Finnemore y Susan K. Sell, op. cit; Kenneth W. Abbott y Duncan Snidal, "The Governance Triangle: Regulatory Standards Institutions and the Shadow of the State", op. cit.; Kenneth W. Abbott y Duncan Snidal, "International Regulation without International Government: Improving IO Performance through Orchestration”, op. cit.; Kenneth Abbott, et al., "Orchestration: Global Governance through Intermediaries", op. cit.; Kenneth W. Abbott, et al., International Organizations as Orchestrators, op. cit.; Kenneth W. Abbott, et al., "Two Logics of Indirect Governance: Delegation and Orchestration”, op. cit.

${ }^{25}$ Deborah D. Avant, Martha Finnemore y Susan K. Sell, op. cit., p. 3.

${ }^{26}$ Kenneth W. Abbott, et al., "Two Logics of Indirect Governance: Delegation and Orchestration", op. cit.

${ }^{27}$ Darren G. Hawkins, et al., Delegation and Agency in International Organizations, Nueva York, Cambridge University Press, 2006, p. 7. 
delegar (actores especializados, capaces de definir los temas en la agenda, monitorear el cumplimiento, resolver disputas, adjudicar decisiones y legitimar o hacer creíbles las políticas que adopta el principal). ${ }^{28}$

La orquestación, en cambio, requiere de intermediarios sobre una base voluntaria. El orquestador carece de controles legales sobre los intermediarios, no puede comandar la cooperación del intermediario, pero al alinearlos, adquiere recursos y capacidades que de otro modo no tendría. ${ }^{29}$ Adicionalmente, gracias a la orquestación, los gobernadores logran incidir en las conductas y políticas de otros.

A continuación, se aplica la categoría analítica de gobernanza indirecta por orquestación al caso de estudio, con la intención de observar la manera en que la oms y la ops interactuaron entre sí, así como la diversidad en los esquemas de coordinación que desarrollaron en sus relaciones con otros agentes.

\section{LA OMS Y LA OPS COMO GOBERNADORES}

La oms y la ops se entienden como gobernadores en el proceso de respuesta a la pandemia de la Covid-19, con capacidad de ejercer poder y afectar políticas de manera transnacional. ${ }^{30}$ En este apartado, se explican las interacciones entre estas organizaciones y el ambiente en el que operan.

La relación entre la oms y la ops es sui generis, pues contempla una combinación de integración formal y de autonomía, o lo que la literatura interorganizacional conceptualiza como instituciones anidadas, ${ }^{31}$ esto es, organizaciones que se

${ }^{28}$ Kenneth W. Abbott, et al., op. cit., p. 723.

29 Ibid., p. 724.

30 Deborah D. Avant, Martha Finnemore y Susan K. Sell, op. cit., p. 2.

31 Spyros Blavoukos y Dimitris Bourantonis, "Nested Institutions", en Rafael Biermann y Joachim A. Koops (eds.), Palgrave Handbook of Inter-Organizational Relations in World Politics, Londres, Palgrave Macmillan, 2017, pp. 303-315. 
encuentran incrustadas una dentro de otra, como las muñecas rusas. ${ }^{32}$ Nacieron como organizaciones independientes, con sus propias estructuras y formas de gobernanza, equidistantes en el tiempo por un periodo de más de cuarenta años. La ops fue creada en 1902 como una organización regional, mientras que la oms nació en 1948 como agencia especializada de las Naciones Unidas. Se unieron en 1949, tras un complejo proceso de negociación. ${ }^{33}$

De conformidad con el acuerdo de su creación, la ops -originalmente conocida como Buró Sanitario Panamericano-, se convirtió en la oficina regional de la oms para las Américas, conservando su nombre e identidad, así como la facultad de adoptar y promover convenciones y programas para el hemisferio occidental, siempre y cuando fuesen compatibles con las políticas y programas de la oms y se financiaran de manera separada. ${ }^{34}$ El capítulo xi de la Constitución de la oms también estipula que cada oficina regional puede establecer sus propias reglas, si bien sus funciones y tareas deben alinearse con las de la oms. ${ }^{35}$

32 Karen J. Alter y Sophie Meunier, "Nested and Overlapping Regimes in the Transatlantic Banana Trade Dispute", Journal of European Public Policy, vol. 13, núm. 3, 2006, p. 363.

33 Marcos Cueto, "The History of International Health: Medicine, Politics, and Two Socio-Medical Perspectives, 1851 to 2000", en Colin McInnes, Kelley Lee y Jeremy Youde (eds.), The Oxford Handbook of Global Health Politics, Oxford, Oxford University Press, 2018, pp. 19-37; Marcos Cueto, Theodore M. Brown y Elizabeth Fee, The World Health Organization. A History, Nueva York, Cambridge University Press, 2019; Liliana De Lima y Eduardo Bruera, "The Pan American Health Organization: Its Structure and Role in the Development of a Palliative Care Program for Latin America and the Caribbean", Journal of Pain and Symptom Management, vol. 20, núm. 6, 2000, pp. 440-448.

${ }^{34}$ Organización Mundial de la Salud/Organización Panamericana de la Salud, oms/ops, Agreement between the World Health Organization and the Pan American Sanitary Organization, A2/VR/1O, Washington D.C., 24 de mayo de 1949, p. 887.

${ }^{35}$ Organización Mundial de la Salud, oms, Constitución de la Organización Mundial de la Salud, Washington D.C., 22 de junio de 1946. 
La incrustación de la ops dentro de la oms -y otras entidades regionales-, organización independiente y previamente consolidada, generó un ambiente operativo complejo marcado por una estructura altamente descentralizada, integrada por seis oficinas regionales semiautónomas, que eligen a su director(a) general, contratan a su propio personal y financian sus programas con donaciones voluntarias de países y fundaciones privadas. ${ }^{36} \mathrm{En}$ el caso de la ops, la descentralización se refuerza además por el hecho de que funciona también como agencia especializada del sistema interamericano, encabezado por la Organización de Estados Americanos (oEA) y, aunque en teoría goza de total autonomía técnica, en la práctica está obligada a tomar en cuenta las recomendaciones de su Asamblea General y sus Consejos. ${ }^{37}$

La descentralización y el ambiente anidado abren así retos de coordinación entre estos gobernadores pues, como sostienen sus directivos, enfrentan problemas para priorizar sus objetivos. ${ }^{38}$ Los esquemas de financiamiento, por ejemplo, dan lugar a pensar en dos organizaciones separadas, pues mientras la oms se financia con contribuciones señaladas (o cuotas fijas de los países miembros) y contribuciones voluntarias, la ops se financia mediante contribuciones señaladas, contribuciones voluntarias y una cuota que le asigna la propia oms. ${ }^{39}$ Entonces, la relación entre los gobernadores está mar-

${ }^{36}$ Elizabeth Fee, Marcos Cueto y Theodore M. Brown, "At the Roots of the World Health Organization's Challenges: Politics and Regionalization", American Journal of Public Health, vol. 106, núm. 111, 2016, pp. 1912-1916.

37 Organización de Estados Americanos, OEA, Carta de la Organización de Estados Americanos, Bogotá, 1948; Organización de Estados Americanos/ Organización Panamericana de la Salud, OEA/ops, "Acuerdo entre el Consejo de la oea y el Consejo Directivo de la Organización Sanitaria Panamericana", OEA/Ser. D/V 3/50, Washington D.C, 23 de mayo de 1950.

${ }^{38}$ Blavoukos Spyros y Dimitris Bourantonis, op. cit.

${ }^{39} \mathrm{El}$ presupuesto de la oms para el bienio 2018-2019 fue de $5.84 \mathrm{bi}-$ llones de dólares y el de la ops de 650 millones de dólares, de los cuales 215.8 corresponden a la asignación presupuestaria de la oms, lo que equivale a $34.8 \%$. Organización Mundial de la Salud, oms, Programme Budget 2020-2021, Ginebra, 2019; Organización Panamericana de la Salud, ops, 
cada por la descentralización y la dificultad para que la oms pueda pronunciarse y actuar como una sola organización, "no dos como sugieren sus mecanismos de financiamiento, o seis como sugiere su estructura regional". ${ }^{40}$

Sin embargo, como se verá en el siguiente apartado, gracias a su conocimiento técnico especializado y a su capacidad para establecer categorías de acción, las dos organizaciones asumieron posiciones de liderazgo y coliderazgo en la gestión de crisis, coordinando de manera voluntaria, y no jerárquica, a muchos otros agentes.

\section{MODALIDADES Y MECANISMOS DE GOBERNANZA INDIRECTA ADOPTADOS POR LA OMS Y LA OPS}

De conformidad con el Reglamento Sanitario Internacional (RSI), el mandato de la oms es "actuar en la contención, control y seguimiento de brotes epidemiológicos y de enfermedades transmisibles" ${ }^{41}$ mediante la ejecución de dos tareas esenciales: investigación y monitoreo. Adicionalmente, al formar parte del sistema internacional humanitario, la oms y la ops están mandatadas por el Comité Permanente entre Organismos (IASC) para asumir funciones de liderazgo y coliderazgo en el clúster en salud.

Un rasgo importante en la ejecución de estos mandatos es que las or necesitan la colaboración activa de los Estados. Son los Estados quienes, en primer instancia, deben reportar, notificar, verificar y responder a brotes y emergencias derivadas de enfermedades transmisibles (aunque actores no gubernamentales puedan reportarlos también). ${ }^{42}$ Las organizaciones

\footnotetext{
"Presupuesto por Programas de la ops 2020-2021", Informe CD57-OD358, 5 de septiembre de 2019.

40 Organización Mundial de la Salud, oms, Gro Harlem Brundtland, Speech to the Fifty-first World Health Assembly, A51/DIV/6, 13 de mayo de 1998. Véase también Blavoukos Spyros y Dimitris Bourantonis, op. cit.

41 Artículo 2 del Rsi, Ginebra, 2005.

42 Marieke, Louis, “¿Para qué sirve la Organización Mundial de la
} 
no tienen mecanismos coercitivos para sancionarlos por incumplimiento, ni los recursos para obligarlos a cooperar, de modo que, por lo general, se ven obligadas a buscar mecanismos alternativos para coordinarlos, impulsar sus recomendaciones y ejecutar sus políticas.

Como veremos a continuación, en el cumplimiento de sus funciones de crisis, la oms y la ops establecieron algunos mecanismos diferenciados de vinculación y coordinación con otros actores que son: (i) el Sistema de Gestión de Incidentes (SGI); (ii) la Red Mundial de Alerta y Respuesta ante brotes epidémicos (Goarn); (iii) el clúster de salud y (iv) la asistencia técnica transversal. Cada uno de estos mecanismos implica diferentes responsabilidades y relaciones de autoridad entre los gobernadores y otros actores (nacionales, organizacionales, subnacionales, humanitarios, privados, etcétera).

Así entonces, respondiendo a las directrices establecidas en el Plan Estratégico de Preparación y Respuesta a la Covid-19 de la oms, de febrero de 2020, el Sistema de Gestión de Incidentes posibilitó una estructura de coordinación multinivel (global, regional, nacional), mediante el establecimiento temporal de unidades de comando. En las Américas, se activaron equipos de apoyo para la gestión de incidentes en 27 oficinas regionales y, desde allí, se coordinaron (hacia arriba) con la oms y (hacia abajo) con equipos de países integrados por las autoridades nacionales de salud y los encargados nacionales y regionales de regulación sanitaria. ${ }^{43}$ El objetivo

Salud? Entrevista a Auriane Guilbaud”, Blog de Nueva Sociedad, abril de 2020, https:/ / nuso.org/articulo/para-que-sirve-la-organizacion-mundial-de-lasalud/ (consulta del 10 de enero de 2021).

${ }^{43} \mathrm{La}$ oms y la ops establecieron y capacitaron a los funcionarios en el manejo de diversas plataformas tecnológicas de información y monitoreo. Por ejemplo, establecieron un tablero de control y datos epidemiológicos de actualización diaria (https://www.esri.com/en-us/covid-19/overview); un mapa interactivo de casos acumulados notificados por países y territorios (https://ais.paho.org/phip/viz/COVID-19EpiDashboard.aspv); ejercicios de simulación para estimar la reproducción del virus y proyectar nuevas infecciones (Covid sım y EpiEstim); y un instrumento para localización y seguimiento de casos (GoData). 
fundamental de estos equipos era prevenir el colapso de los sistemas de salud ante la escalada de contagios por Covid-19, apoyando a los gobiernos en (i) la identificación de necesidades y la elaboración de planes de respuesta; (ii) la colecta de información actualizada sobre la evolución y comportamiento de la Covid-19, útil para ajustar o reformular la respuesta y, (iii) la elaboración de recomendaciones para el fortalecimiento y protección del sector sanitario. ${ }^{44}$

De esta manera, gracias al manejo de información actualizada, basada en evidencia científica, y en el monitoreo sistemático del fenómeno, ${ }^{45}$ la oms y la ops adquirieron autoridad para participar en la elaboración y el seguimiento de políticas y planes nacionales de respuesta. Ello, sin embargo, no quiere decir que pudiesen incidir directamente en la implementación de dichas políticas o asumiesen las responsabilidades de los gobiernos. Antes bien, cada gobierno interpretó la información y adoptó, o no, las recomendaciones que le parecieron más convenientes. La coordinación por orquestación aquí fue algo similar a una "red centralizada" ${ }^{46}$ donde las or-

${ }^{44}$ Organización Panamericana de la Salud, ops, "Informes de situación de la COVID-19", 2020, https://www.paho.org/es/informes-situacioncovid-19 (consulta del 18 de enero de 2021); Naciones Unidas, Asamblea General, Strengthening of the Coordination of Emergency Humanitarian Assistance of the United Nations, Resolución 42/182, 19 de diciembre de 1991; Organización Mundial de la Salud, oms, "2019 Novel Coronavirus (2019nCoV): Strategic Preparedness and Response Plan", 2020, https://www. who.int/docs/default-source/coronaviruse/srp-04022020.pdf (consulta del 20 de febrero de 2021); Organización Mundial de la Salud, oms, "Coronavirus Disease (COVID-19) Dashboard”, 2020, https://covid19.who. int/ (consulta del 20 de febrero de 2021); Organización Mundial de la Salud, oms, "Covid 19 Strategy Update", 14 de abril de 2020, https:// www.who.int/publications/m/item/covid-19-strategy-update (consulta del 20 de febrero de 2021).

${ }^{45}$ Chris Ansell y Ann Keller, Adapting the Incident Command Model for Knowledge-Base Crisis: The Case of the Centers for Disease Control and Prevention, Berkeley, University of California, Collaboration Series, 2014.

${ }^{46}$ Donald P. Moynihan, "The Network Governance of Crisis Response: Case Studies of Incident Command Systems", Journal of Public Administration Research and Theory, vol. 19, núm. 4, 2009, p. 896. 
ganizaciones actuaron como nodos, desde los cuales se distribuía información valiosa sobre el fenómeno y se buscaba adecuar la respuesta a las condiciones particulares de los diversos actores involucrados.

Otro mecanismo de coordinación por orquestación es la Red Mundial de Alerta (GOARN), encargada de proveer información y alerta temprana ante brotes epidemiológicos. Esta red posibilitó la vinculación de la oms y la ops con comunidades epistémicas y de investigación científica. Así, por ejemplo, durante la pandemia, la red proporcionó información científica, altamente relevante para comprender la naturaleza del coronavirus, su origen, su severidad y sus mecanismos de transmisión. A partir de esta información se diseñaron protocolos, guías técnicas y recomendaciones para el manejo clínico de los enfermos, su aislamiento, la aplicación de pruebas y el desarrollo de tratamientos y vacunas. ${ }^{47}$ Bajo esta forma de coordinación, las organizaciones adquirieron recursos y capacidades orientadas al control efectivo de la pandemia, si bien ello pudiera tener implicaciones en cuanto a compaginar intereses privados y lógicas de mercado con la provisión de bienes públicos globales.

A nivel global, la oms y la ops se vincularon con más de 230 diferentes instituciones técnicas y operativas (153 instituciones y 37 redes adicionales) que incluyen agencias de gobierno, instituciones público-privadas de salud globales, regionales y nacionales, centros especializados en epidemiología, control de infecciones, ciencias biomédicas, redes de laboratorios y organismos de las Naciones Unidas que realizan tareas de identificación, confirmación y respuesta a brotes epidémicos de importancia internacional. ${ }^{48}$ A nivel regional, las or se vincularon con 14 socios y cuatro redes adicionales entre los que se encuentran la Agencia de Salud Pública del

${ }^{47}$ Evan S. Michelson, "Dodging a Bullet: who, saRs, and the Successful Management of Infectious Disease", Bulletin of Science, Technology and Society, vol. 25, núm. 5, 2005, pp. 379-386.

${ }^{48}$ John S. Mackenzie, et al., "The Global Outbreak Alert and Response Network", Global Public Health, vol. 9, núm. 9, 2014, p. 1024. 
Caribe (Trinidad y Tobago), el Centro Nacional de Epidemiología (Guatemala), la Dirección General de Epidemiología de la Secretaría de Salud (México), el Institut Pasteur (Guyana), el Instituto Evandro Chagas (Brasil), el Instituto Nacional de Enfermedades Virales Humanas J. Maiztegui (Argentina), el Instituto Nacional de Salud (Perú), el ProEpi y la Universidad Federal de Río de Janeiro (Brasil). La red fortalece a la oms y la ops en el cumplimiento de sus funciones sustantivas de gestión de crisis -investigación y monitoreo del fenómeno-, de manera que, para algunos analistas, se está convirtiendo en el brazo operativo del RSI. ${ }^{49}$

El tercer mecanismo de coordinación por orquestación es el clúster de salud. El clúster forma parte del sistema de respuesta humanitaria de las Naciones Unidas, y está regulado por el Comité Permanente (IASC) y por el Coordinador Humanitario de la Oficina de Coordinación de Asuntos Humanitarios (ОСНА). Los clústeres humanitarios se activan en las fases tempranas de emergencias para proveer coordinación sectorial temporal (salud, albergue, protección, agua, sanidad e higiene, etcétera) ${ }^{50} \mathrm{y}$ vinculan tanto a las agencias especializadas de las Naciones Unidas como a las organizaciones no gubernamentales (ONG) internacionales y locales. Dentro del clúster, cada una de las organizaciones participantes contribuye a la

49 Ibid, p. 1026.

${ }^{50}$ A escala global existen once clústeres: (1) Salud, (2) Nutrición, (3) Protección, (4) Refugio, (5) Agua, sanidad e higiene, (6) Coordinación y administración de campamentos, (7) Recuperación temprana, (8) Educación, (9) Seguridad alimentaria, (10) Telecomunicaciones de emergencia y (11) Logística. Oficina de las Naciones Unidas para la Coordinación de Asuntos Humanitarios, осHA, "What is the Cluster Approach?", осHA Services/Humanitarian Response, 2019, https://www.humanitarianrespo nse.info/en/coordination/clusters/what-cluster-approach (consulta del 17 de noviembre de 2020); Oficina de las Naciones Unidas para la Coordinación de Asuntos Humanitarios, OCHA, "Inter Cluster Coordination", осна Services/Humanitarian Response, 2019, https://www.humanitarianresponse.info/en/how-to/do-inter-cluster-coordination\#: :text= Inter\%2Dcluster\% 20coordination \% 20takes $\% 20$ place,support $\% 20$ of $\% 20$ the\%20national\%20response (consulta del 17 de noviembre de 2020). 
identificación de necesidades y ejecución de la respuesta evitando tanto la duplicidad como los vacíos y asegurando la aplicación de estándares internacionales sobre derechos humanos. ${ }^{51}$ Se integra por una agencia líder y diversos actores humanitarios, en una estructura de coordinación horizontal que puede entenderse como una red "flojamente acoplada", ${ }^{52}$ donde los participantes contribuyen con información y propuestas para elaborar planes de respuesta, pero no están obligados a seguirlos y aplicarlos si no lo desean.

Durante la pandemia de Covid-19 y en seguimiento al Plan Global de Respuesta Humanitaria, осHA identificó 63 países particularmente vulnerables a la pandemia, debido a que en ellos ya se vivían crisis humanitarias previas, con Colombia, Haití y Venezuela como los países señalados en la región. Debido a sus bajos índices de desarrollo, crecimiento económico y débiles sistemas de salud, así como a las problemáticas derivadas de conflicto civil, altos niveles de pobreza, problemas de inseguridad alimentaria o recepción masiva de refugiados, migrantes y desplazados, se decidió activar el clúster de salud en estos tres países. ${ }^{53}$

Para atender a estas poblaciones en condición de doble riesgo -humanitario y por la Covid-19-, se hizo un requerimiento de 6.710 millones de dólares. ${ }^{54}$ De éstos, se estimó que 406.7 millones se destinarían a cubrir las necesidades

51 Comité Permanente entre Organismos (IASC), "Operational Guidance on Responsibilities of Cluster/ Sector Leads and OCHA in Information Management", IAscTask Force on Information Management, 2008; Comité Permanente entre Organismos (IASC), "About the Inter-Agency Standing Committee", 2020, https://interagencystandingcommittee.org/the-interagency-standing-committee (consulta del 17 de noviembre de 2020).

${ }^{52}$ Karl E. Weik, "Educational Organizations as Loosely Coupled Systems", Administrative Science Quarterly, vol. 21, núm. 1, 1976, pp. 1-19.

53 Oficina de las Naciones Unidas para la Coordinación de Asuntos Humanitarios (OCHA), "Plan Global de Respuesta Humanitaria Covid-19 abril-diciembre 2020", 2020, https://www.unocha.org/sites/unocha/files /GHRP-COVID19_MayUpdate_Abridged_SPA.pdf (consulta del 18 de enero de 2021).

54 Ibid., p. 3. 
humanitarias en Colombia; 424.3 millones a Haití y 750.0 millones a Venezuela para apoyar su Plan de Respuesta Humanitaria preexistente, más 1.440 millones para apoyar el Plan de Respuesta para Refugiados y Migrantes. ${ }^{55}$ Así, en Venezuela, con el apoyo del gobierno de Nicolás Maduro, se activaron ocho clústeres humanitarios, donde participaron 116 organizaciones operacionales (59 ONG nacionales y locales; 19 internacionales, 12 instituciones públicas y ocho agencias de la ONU). Esta estructura de coordinación alcanzó a 24 estados y dependencias federales, 335 municipios y 3.7 millones de personas en noviembre de $2020{ }^{56}$ En Colombia, el clúster integró a seis agencias de la ONU, 25 ONG internacionales y cinco ONG nacionales. ${ }^{57}$ Así, en la vinculación con los actores humanitarios, la oms y la ops asumen el liderazgo de la respuesta, sin que ello signifique que tienen comando jerárquico o capacidad de control sobre otros actores. Su función es servir de puente entre los actores para cruzar información y trazar una ruta operativa.

Finalmente, de manera transversal a toda su actividad, la OMs y la OPs actuaron como burocracias racionales, proveedoras de asistencia y conocimiento técnico experto. Durante la pandemia, este tipo de conocimiento fue clave para que las dos organizaciones pudieran elaborar recomendaciones y diseñar categorías de acción dirigidas a estructurar la respuesta de los gobiernos, pero también buscaron socializarla con otros actores subnacionales involucrados en la respuesta y público en general. Así, impartieron más de 206 cursos virtuales de entrenamiento y capacitación, publicaron alrededor de 109 guías técnicas y diseñaron cientos de protocolos e info-

${ }^{55}$ Ibid., p. 6.

${ }^{56}$ Oficina de las Naciones Unidas para la Coordinación de Asuntos Humanitarios, OCHA, "Informe de situación sobre Venezuela", noviembre de 2020, https:/ / www.unocha.org/venezuela (consulta del 20 de noviembre de 2020).

${ }^{57}$ Organización Panamericana de la Salud, ops, "Cluster de salud Colombia: sistema de información”, 2020, https:/ /www.paho.org/es/cluster-sa lud-colombia-sistema-informacion (consulta del 20 de noviembre de 2020). 
grafías para la aplicación de pruebas, detección y rastreo, medidas preventivas de contagio, manejo clínico, medidas de protección, etcétera, ${ }^{58}$ así como medidas de confinamiento social, hábitos personales y uso de mascarillas. ${ }^{59}$ Este proceso de capacitación y entrenamiento, más las conferencias de prensa diarias, sirvió entonces para familiarizar a la ciudadanía con principios, estándares y recomendaciones. Adicionalmente, ofrecieron asesoría y asistencia técnica a los gobiernos latinoamericanos y caribeños en torno al mercado de compra y al transporte para insumos médicos, respiradores y equipos de protección. Con este propósito, enviaron 165 equipos médicos de emergencia a 21 países de la región, donde además contribuyeron a la instalación de 209 centros de asistencia alternativos (en estadios, bases militares, estaciones de bomberos, hoteles, etcétera). ${ }^{60} \mathrm{Al}$ ofrecer asistencia y capacitación técnica, las organizaciones se relacionaron con actores subnacionales, asociaciones de profesionistas, sector privado, medios de comunicación y ciudadanos, lo que amplió su rango de acción y capacidades.

Con base en lo analizado, es posible asumir que la oms y la ops alcanzaron algunos buenos resultados en la respuesta. Para octubre de 2020 se reportó que de un total de 54 países y territorios americanos:

58 Organización Panamericana de la Salud, ops, "Informes de situación de la COVID-19", 2020, https:/ / www.paho.org/es/informes-situacioncovid-19 (consulta del 20 de noviembre de 2020).

${ }^{59}$ Organización Panamericana de la Salud, ops, "Informes de situación de la COVID-19", 2020, https://www.paho.org/es/informes-situacioncovid-19 (consulta del 20 de noviembre de 2020); Organización Panamericana de la Salud, ops, "COVID-19 Information System for the Region of the Americas", 2020, https:/ / paho-covid19-response-who.hub.arcgis.com/ (consulta del 20 de noviembre de 2020).

${ }^{60}$ Chile (28), Ecuador (23) y Perú (52) fueron los países que recibieron el mayor número de equipos médicos de emergencia y, Chile y Guayana (17), Ecuador (26), México (91), Perú (16) y Trinidad y Tobago (12) los países donde se instalaron más centros de asistencia alternativos. Organización Panamericana de la Salud, ops, "PAHO's COVID-19 EMT Response", Covid-19 PAHO/WHO Response Report, núm. 33, 9 de noviembre de 2020. 
- 32 lograron elaborar planes de preparación y respuesta;

- 35 ajustaron planes y políticas con base en la información actualizada provista por las organizaciones;

- 35 activaron mecanismos intersectoriales de respuesta;

- 38 construyeron capacidades para detección y diagnóstico mediante pruebas moleculares;

- 51 establecieron sistemas de diagnóstico;

- 20 establecieron sistemas de monitoreo y vigilancia de infecciones respiratorias agudas graves y pseudogripe de la Covid-19 y;

- 23 comenzaron a implementar sistemas de detección y vigilancia con Go Data. ${ }^{61}$

En suma, los mecanismos de coordinación aplicados por la oms y la ops para responder a las funciones de crisis conferidas en su mandato, ofrecen evidencia de su papel en el desarrollo de la gobernanza indirecta por orquestación. Mediante el SGI, las organizaciones se vincularon, desde oficinas regionales, con gobiernos nacionales, autoridades sanitarias, agencias reguladoras, hospitales, laboratorios, centros de atención y, en general, con los actores de primera línea de respuesta, para apoyarlos en la identificación de necesidades y riesgos. Actuaron en esquemas de coordinación sin jerarquía o control formal de los participantes, pero con capacidad de incidir gracias al "control burocrático de información".

Mediante la Red de Alerta, interactuaron con institutos de investigación públicos y privados, farmacéuticas, universidades y centros especializados en procesos de investigación médica y científica, orientadas al desarrollo tecnológico para el control del brote. La vinculación con todos estos actores aportó conocimiento científico sobre la naturaleza, evolución y el manejo del fenómeno, y sumó recursos y capacidades de acción que, de otra manera, las organizaciones no tendrían.

${ }^{61}$ Organización Panamericana de la Salud, ops, "Respuesta de la Organización Panamericana de la Salud a la COVID-19 en la región de las Américas, del 17 de enero al 31 de mayo del 2020", 10 de julio de 2020, https://iris.paho.org/handle/10665.2/52454 (consulta del 20 de noviembre de 2020). 


\section{CuAdro 1}

Mecanismos de coordinación y orquestación de la respuesta aplicados por la OMS-OPS

\begin{tabular}{|c|c|c|c|c|}
\hline $\begin{array}{l}\text { Mecanismo de } \\
\text { coordinación }\end{array}$ & $\begin{array}{l}\text { Objetivo del } \\
\text { mecanismo }\end{array}$ & $\begin{array}{c}\text { Naturaleza de la } \\
\text { vinculación }\end{array}$ & $\begin{array}{c}\text { Fortalezas de la } \\
\text { orquestación }\end{array}$ & $\begin{array}{l}\text { Retos de la } \\
\text { orquestación }\end{array}$ \\
\hline $\begin{array}{l}\text { Sistema de } \\
\text { Gestión de } \\
\text { Incidentes }\end{array}$ & $\begin{array}{l}\text { Estructura de } \\
\text { coordinación } \\
\text { multinivel } \\
\text { mediante } \\
\text { equipos } \\
\text { regionales y } \\
\text { nacionales de } \\
\text { respuesta. }\end{array}$ & $\begin{array}{l}\text { Oficinas regionales } \\
\text { de oms-ops, } \\
\text { grupos nacionales } \\
\text { de respuesta } \\
\text { (ministerios } \\
\text { de gobierno } \\
\text { y de salud, } \\
\text { organizaciones } \\
\text { sanitarias públicas } \\
\text { y privadas), } \\
\text { organizaciones } \\
\text { regionales y locales. }\end{array}$ & $\begin{array}{l}\text { Impulsar una } \\
\text { respuesta común } \\
\text { entre múltiples } \\
\text { actores, en diversos } \\
\text { niveles de auto- } \\
\text { ridad, mediante } \\
\text { información, } \\
\text { asesoría y } \\
\text { monitoreo dirigido } \\
\text { a evitar el colapso } \\
\text { de los sistemas de } \\
\text { salud. }\end{array}$ & $\begin{array}{l}\text { No están facultados } \\
\text { para intervenir en } \\
\text { la implementación } \\
\text { de los planes } \\
\text { de respuesta. } \\
\text { Los gobiernos } \\
\text { interpretan la } \\
\text { información y } \\
\text { aceptan o no las } \\
\text { recomendaciones. }\end{array}$ \\
\hline Red de alerta & $\begin{array}{l}\text { Estructura de } \\
\text { coordinación } \\
\text { con comunida- } \\
\text { des epistémicas } \\
\text { que poseen } \\
\text { recursos } \\
\text { científicos y } \\
\text { tecnológicos } \\
\text { especializados. }\end{array}$ & $\begin{array}{l}\text { Centros de } \\
\text { investigación } \\
\text { públicos y privados, } \\
\text { farmacéuticas, } \\
\text { laboratorios, } \\
\text { fundaciones, } \\
\text { universidades, } \\
\text { etcétera. }\end{array}$ & $\begin{array}{l}\text { Incidir en los } \\
\text { procesos de } \\
\text { investigación y } \\
\text { evolución del } \\
\text { brote, así como } \\
\text { en mecanismos } \\
\text { de control } \\
\text { (tratamientos y } \\
\text { vacunas). }\end{array}$ & $\begin{array}{l}\text { La vinculación } \\
\text { con estos actores } \\
\text { abre la puerta a la } \\
\text { participación del } \\
\text { sector privado y } \\
\text { actores de mercado } \\
\text { en la provisión de } \\
\text { bienes públicos } \\
\text { globales. }\end{array}$ \\
\hline $\begin{array}{l}\text { Clúster } \\
\text { humanitario } \\
\text { de salud }\end{array}$ & $\begin{array}{l}\text { Estructura de } \\
\text { coordinación } \\
\text { sectorial } \\
\text { temporal } \\
\text { mediante redes } \\
\text { de actores } \\
\text { humanitarios } \\
\text { que comparten } \\
\text { objetivos. }\end{array}$ & $\begin{array}{l}\text { Agencias especia- } \\
\text { lizadas del sistema } \\
\text { de las Naciones } \\
\text { Unidas, ONG } \\
\text { internacionales } \\
\text { y locales, actores } \\
\text { humanitarios, } \\
\text { etcétera. }\end{array}$ & $\begin{array}{l}\text { Asistir a grupos vul- } \\
\text { nerables, evitando } \\
\text { vacíos o duplicida- } \\
\text { des en la respuesta } \\
\text { y aplicando están- } \\
\text { dares internacio- } \\
\text { nales de derechos } \\
\text { humanos. }\end{array}$ & $\begin{array}{l}\text { Lograr la } \\
\text { coordinación } \\
\text { entre los clústeres } \\
\text { (salud, educación, } \\
\text { agua etcétera) } \\
\text { y en el interior } \\
\text { de los mismos } \\
\text { con actores no } \\
\text { gubernamentales. } \\
\text { Aunque sostienen } \\
\text { los mismos } \\
\text { objetivos se genera } \\
\text { competencia. }\end{array}$ \\
\hline
\end{tabular}

Fuente: elaboración propia con base en el análisis organizacional y la revisión de documentos y reportes primarios de las organizaciones. 
La red con comunidades epistémicas, al igual que con actores humanitarios internacionales y locales en torno a los clústeres de salud, ofrecen mecanismos de orquestación en forma de redes horizontales "flojamente acopladas", donde las agencias líderes (oms y OPs) no tienen autoridad o capacidad para imponerse sobre otras agencias u organizaciones participantes pero que, al coordinarse, logran mejores resultados, pues evitan la duplicidad o el vacío en la respuesta ${ }^{62}$ Estas redes funcionaron gracias a que las comunidades epistémicas y los actores humanitarios comparten objetivos, principios y estándares de acción.

Finalmente, la cualidad burocrática de las organizaciones, específicamente su autoridad racional legal y conocimiento experto, sirvió para que establecieran mecanismos de acción y socializaran procedimientos, normas y estándares para el manejo y monitoreo del coronavirus, alcanzando incluso a actores subnacionales y al público en general. En el cuadro que aparece a continuación se hace un resumen de los mecanismos de coordinación que dieron forma a la orquestación y se mencionan algunas de sus fortalezas y retos.

\section{Conclusiones}

El estudio de los mecanismos de gobernanza indirecta mediante orquestación aplicados por organizaciones regionales e internacionales en contextos de crisis global es todavía terra incognita. ${ }^{63}$ Aunque la bibliografía sobre gestión de crisis sostiene que la capacidad organizacional es un elemento clave para facilitar la coordinación emergente de múltiples actores

${ }^{62}$ Max Jr. Stephenson, "Making Humanitarian Relief Networks More Effective: Operational Coordination, Trust and Sense Making", Disasters, vol. 29, núm. 4, 2005, pp. 337-350; J. Douglas Orton y Karl E. Weick, "Loosely Coupled Systems: A Reconceptualization", The Academy of Management Review, vol. 15, núm. 2, 1990, pp. 203-223.

${ }^{63}$ Arjen Boin, op. cit., p. 295. 
de manera transnacional, ${ }^{64}$ los procesos de coordinación organizacional siguen siendo difíciles de lograr en escenarios caracterizados por la descentralización de las decisiones.

El artículo buscó analizar los mecanismos de coordinación, orquestados por la oms y la ops, que fomentaron esquemas de gobernanza indirecta, con sus fortalezas y retos. Lo que observamos es que las organizaciones vincularon a muy diversos agentes en sus procesos de respuesta y, al hacerlo, confeccionaron formas de coordinación diferenciada: en algunos casos se trató de coordinación sectorial, como en el clúster de salud, y en otras implicó una coordinación multinivel como en el SGI. En ocasiones se desarrollaron redes horizontales con liderazgo predefinido y, en otras, redes "flojamente acopladas", donde actuaron como nodos claves de información y conocimiento. La combinación de estos mecanismos permite argumentar que las organizaciones participan en distintos momentos y con diversas capacidades en el proceso de gobernanza transnacional.

Sin embargo, los mecanismos de coordinación por orquestación permiten observar también que, en contextos de incertidumbre y fragmentación de la respuesta, se requieren algunos mínimos de coordinación por jerarquía y centralización, de allí que estos mecanismos exhiban algunas combinaciones extrañas de acción horizontal en red y, al mismo tiempo, con formas de liderazgo o centralización.

Por otro lado, los mecanismos de coordinación por orquestación son claramente instrumentos de intervención no coercitivos y ello tiene implicaciones en materia de cumplimiento, pues no están diseñados para comandar, controlar o dirigir a los actores en sus interacciones. Como instrumentos de coordinación, tienen potencial para establecer categorías de acción, fijar significados y socializar normas y procedimientos a favor de una respuesta más o menos homogénea,

${ }^{64}$ Donald Blondin y Arjen Boin, "Cooperation in the Face of Transboundary Crisis: A Framework for Analysis", Perspectives on Public Management and Governance, vol. 3, núm. 3, 2020, pp. 197-209. 
brincando incluso la intermediación gubernamental y llegando a actores subnacionales. ${ }^{65}$ Sin embargo, también enfrentan el enorme reto de abrir la respuesta a muy diversos actores, con lógicas, intereses y dinámicas de acción diferentes, como sucede con los actores privados y sus lógicas de mercado. De manera que, en ocasiones, resulta complicado saber quién toma las decisiones y con qué objetivos.

Adicionalmente, el artículo buscaba contribuir al debate teórico de la gobernanza transnacional, mediante el análisis de un caso empírico. Al respecto, se identificaron cuatro mecanismos de coordinación por orquestación, con variaciones en los tipos de actores que se vincularon, las relaciones de autoridad y las dinámicas de control burocrático. Ello permite argumentar que la coordinación mediante orquestación no responde a un solo modelo y se materializa con estructuras y formas organizacionales sumamente diversas, desde las cuales los actores organizacionales participan como agentes activos y autoritativos, contribuyendo a la gobernanza desde diversos frentes. ${ }^{66}$

\section{REFERENCIAS BIBLIOGRÁFICAS}

Аввотт, Kenneth W. y Duncan Snidal, "International Regulation without International Government: Improving IO Performance through Orchestration", Review of International Organizations, vol. 5, núm. 3, 2010, pp. 315-344.

Аввотт, Kenneth W. y Duncan Snidal, "The Governance Triangle: Regulatory Standards Institutions and the Shadow of the State", en Walter Mattli y Ngaire Woods (eds.), The Politics of Global Regulation, Princeton, University Press, 2009, pp. $44-88$.

${ }^{65}$ Giovanni Agostinis y Stefano Palestini, "Transnational Governance in Motion: Regional Development Banks, Power Politics, and the Rise and Fall of South America's Infrastructure Integration", Governance, 2020, p. 4. ${ }^{66}$ Deborah D. Avant, Martha Finnemore y Susan K. Sell, op. cit. 
Аввотт, Kenneth W., Philipp Genschel, Duncan Snidal y Bernhard ZANGL, "Two Logics of Indirect Governance: Delegation and Orchestration", British Journal of Political Science, vol. 46, núm. 4, 2016, pp. 719-729.

Аввотт, Kenneth W., Philipp Genschel, Duncan Snidal y Bernhard ZANGL (eds.), International Organizations as Orchestrators, Cambridge, University Press, 2015.

Аввотт, Kenneth W., Philipp Genschel, Duncan Snidal y Bernhard ZANGL (eds.), "Orchestration: Global Governance through Intermediaries”, 2012, http:/ /ssrn.com/abstract=2125452 (consulta del 20 de febrero de 2021).

Agostinis, Giovanni y Stefano Palestini, “Transnational Governance in Motion: Regional Development Banks, Power Politics, and the Rise and Fall of South America's Infrastructure Integration”, Governance, 2020, pp. 1-20, https://doi.org/10.1111/ gove. 12529

Alter, Karen J. y Sophie Meunier, "Nested and Overlapping Regimes in the Transatlantic Banana Trade Dispute", Journal of European Public Policy, vol. 13, núm. 3, 2006, pp. 362-382.

Ansell, Chris y Ann Keller, Adapting the Incident Command Model for Knowledge-Based Crisis: The Case of the Centers for Disease Control and Prevention, Berkeley, University of California, Collaboration Series, 2014.

Avant, Deborah D., Martha Finnemore y Susan K. Sell, Who Governs the Globe?, Cambridge, University Press, 2010.

Axelrod, Robert, The Evolution of Cooperation, Nueva York, Basic Books, 1983.

BARnett, Michael y Martha Finnemore, Rules for the World: International Organizations in Global Politics, Ithaca, Cornell University Press, 2004.

Barnett, Michael y Martha Finnemore, "The Politics, Power and Pathologies of International Organizations", International Organization, vol. 53, núm. 4, 1999, pp. 699-732.

Biermann, Rafael, "Designing Cooperation among International Organizations. Autonomy Concerns, the Dual Consensus Rule, and Cooperation Failure", Journal of International Organizations Studies, vol. 6, núm. 2, 2015, pp. 45-66. 
Blavoukos, Spyros y Dimitris Bourantonis, "Nested Institutions", en Rafael Biermann and Joachim A. Koops (eds.), Palgrave Handbook of Inter-Organizational Relations in World Politics, Londres, Palgrave Macmillan, 2017, pp. 303-315.

Blondin, Donald y Arjen Boin, "Cooperation in the Face of Transboundary Crisis: A Framework for Analysis", Perspectives on Public Management and Governance, vol. 3, núm. 3, 2020, pp. 197-209. Boin, Arjen, "The New World of Crises and Crisis Management: Implications for Policymaking and Research", Review of Policy Research, vol. 26, núm. 4, 2009, pp. 367-377.

Boin, Arjen y Fredrik Bynander, "Explaining Success and Failure in Crisis Coordination," Geografiska Annaler, vol. 97, núm. 1, 2014, pp. 123-135.

Boin, Arjen, Paul 't Hart, Eric Stern y Bengt Sundelius, The Politics of Crisis Management: Public Leadership under Pressure, Cambridge, University Press, 2016.

Christensen, Tom, Per Laegreid y Lisa H. Rykкja, “Organizing for Crisis Management: Building Governance Capacity and Legitimacy", Public Administration Review, vol. 76, núm. 6, 2016, pp. $887-897$.

Comité Permanente entre Organismos (IASC), "About the InterAgency Standing Committee”, 2020, https:/ /interagencystand ingcommittee.org/the-inter-agency-standing-committee (consulta del 17 de noviembre de 2020).

Comité Permanente entre Organismos (IASC), "Operational Guidance on Responsibilities of Cluster/ Sector Leads and OCHA in Information Management", IASC Task Force on Information Management, 2008.

Cueto, Marcos, "The History of International Health: Medicine, Politics, and Two Socio-Medical Perspectives, 1851 to 2000", en Colin McInnes, Kelley Lee y Jeremy Youde (eds.), The Oxford Handbook of Global Health Politics, Oxford, Oxford University Press, 2018, pp. 19-37.

Cueto, Marcos, Theodore M. Brown y Elizabeth Fee, The World Health Organization. A History, Nueva York, Cambridge University Press, 2019. 
De Lima, Liliana y Eduardo Bruera, “The Pan American Health Organization: Its Structure and Role in the Development of a Palliative Care Program for Latin America and the Caribbean", Journal of Pain and Symptom Management, vol. 20, núm. 6, 2000, pp. 440-448.

Fee, Elizabeth, Marcos Cueto y Theodore M. Brown, "At the Roots of the World Health Organization's Challenges: Politics and Regionalization", American Journal of Public Health, vol. 106, núm. 111, 2016, pp. 1912-1916.

Hawkins, Darren G., et al., Delegation and Agency in International Organizations, Nueva York, Cambridge University Press, 2006.

Jervis, Robert, "Cooperation under the Security Dilemma”, World Politics, vol. 30, núm. 2, 1978, pp. 167-214.

Keohane, Robert O., "Reciprocity in International Relations", International Organization, vol. 40, núm. 1, 1986, pp. 1-27.

Keohane, Robert, After Hegemony: Cooperation and Discord in the World Political Economy, Princeton, University Press, 1984.

Krasner, Stephen D. (eds.), International Regimes, Ithaca, Cornell University Press, 1983.

Koops, Joachim A., "Inter-organizational Approaches", en Knud E. Jørgensen y Katie V. Laatikainen (eds.), The Routledge Handbook of Europe and Multilateral Institutions, Londres, Routledge, 2013, pp. 71-85.

Laegreid, Per, Külli Sarapuu, Lise Rykkja y Tina Randma-Litv (eds.), Organizing for Coordination in the Public Sector: Practices and Lessons from 12 European Countries, Basingstoke, Palgrave Macmillan, 2014.

Louis, Marieke, “¿Para qué sirve la Organización Mundial de la Salud? Entrevista a Auriane Guilbaud", Blog de Nueva Sociedad, abril de 2020, https://nuso.org/articulo/para-que-sirve-la-organizacion -mundial-de-la-salud/ (consulta del 10 de enero de 2021).

Mackenzie, John S., et al., "The Global Outbreak Alert and Response Network”, Global Public Health, vol. 9, núm. 9, 2014, pp. 1023-1039.

Mearsheimer, John J., "The False Promise of International Institutions", International Security, vol. 19, núm. 3, 1994-1995, pp. 5-49. 
Michelson, Evan S., "Dodging a Bullet: who, sars, and the Successful Management of Infectious Disease", Bulletin of Science, Technology and Society, vol. 25, núm. 5, 2005, pp. 379-386.

Moynihan, Donald P., "The Network Governance of Crisis Response: Case Studies of Incident Command Systems", Journal of Public Administration Research and Theory, vol. 19, núm. 4, 2009, pp. 895-915.

Naciones Unidas, Asamblea General, Streghtening of the Coordination of Emergency Humanitarian Assistance of the United Nations, Resolución 42/182, 19 de diciembre de 1991.

Najam, Adil, Mihaela Papa y Nadaa Tатуав, Global Environmental Governance: A Reform Agenda, Winnipeg, International Institute for Sustainable Development (IISD), 1999.

Nohrstedt, Daniel, Fredrik Bynander, Charles Parker y Paul 't Hart, "Managing Crisis Collaboratively: Prospect and Problems- A Systematic Literature Review", Perspectives on Public Management and Governance, vol. 1, núm. 4, 2018, pp. 257-271.

Овектнür, Sebastian y Olav Schram Stokke (eds.), Managing Institutional Complexity: Regime Interplay and Global Environmental Change, Cambridge, мiт Press, 2011.

Oestreich, Joel E. (ed.), International Organizations as Self-Directed Actors. A Framework for Analysis, Nueva York, Routledge, 2012.

Oficina de las Naciones Unidas para la Coordinación de Asuntos Humanitarios, осHA, "Plan Global de Respuesta Humanitaria Covid-19 abril-diciembre 2020", 2020, https://www.unocha. org/sites/unocha/files/GHRP-COVID19_MayUpdate_ Abridged_SPA.pdf (consulta del 18 de enero de 2021).

Oficina de las Naciones Unidas para la Coordinación de Asuntos Humanitarios, осHA, "Informe de situación sobre Venezuela", noviembre de 2020, https:/ / www.unocha.org/venezuela (consulta del 20 de noviembre de 2020).

Oficina de las Naciones Unidas para la Coordinación de Asuntos Humanitarios, осHA, "Inter Cluster Coordination", осна Services /Humanitarian Response, 2019, https:/ /www.humanitarianrespo nse.info/en/how-to/do-inter-cluster-coordination\#: :text=In ter $\% 2$ Dcluster $\% 20$ coordination $\% 20$ takes $\% 20$ place,support $\%$ 
20of\%20the\%20national\%20response (consulta del 17 de noviembre de 2020).

Oficina de las Naciones Unidas para la Coordinación de Asuntos Humanitarios, OCHA, "What is the Cluster Approach?", осHA Services/Humanitarian Response, 2019 https://www.humanitari anresponse.info/en/coordination/clusters/what-cluster-ap proach (consulta del 17 de noviembre de 2020).

Olson, Mancur, The Logic of Collective Action: Public Goods and the Theory of Groups, Cambridge, Harvard University Press, 1965.

Olsson, Eva Karin, "Transboundary Crisis Networks: The Challenge of Coordination in the Face of Global Threats", Risk Management, vol. 17, núm. 2, 2015, pp. 91-108.

Olsson, Eva Karin y Bertjan Verbeek, "International Organizations and Crisis Management”, en Bob Reinalda (ed.), Routledge Handbook of International Organization, Nueva York, Routledge, 2013, pp. 324-336.

Organización de Estados Americanos, ozA, Carta de la Organización de Estados Americanos, Bogotá, 1948.

Organización de Estados Americanos/Organización Panamericana de la Salud, oeA/ops, "Acuerdo entre el Consejo de la oeA y el Consejo Directivo de la Organización Sanitaria Panamericana", oEA/Ser. D/V 3/50, Washington D.C., 23 de mayo de 1950.

Organización Mundial de la Salud, oms, "WHO Coronavirus Disease (COVID-19) Dashboard", https://covid19.who.int/ (consulta del 18 de febrero de 2021).

Organización Mundial de la Salud, oms, "2019 Novel Coronavirus (2019-nCoV): Strategic Preparedness and Response Plan”, 2020, https://www.who.int/docs/default-source/coronaviruse/srp-04022020.pdf (consulta del 20 de febrero de 2021).

Organización Mundial de la Salud, oms, "Coronavirus Disease (COVID-19) Dashboard”, 2020, https://covid19.who.int/ (consulta del 20 de febrero de 2021).

Organización Mundial de la Salud, oms, "Covid 19 Strategy Update", 14 de abril de 2020, https:/ /www.who.int/publications/ $\mathrm{m} /$ item/covid-19-strategy-update (consulta del 20 de febrero de 2021). 
Organización Mundial de la Salud, oms, "Programme Budget 20202021”, Ginebra, 2019.

Organización Mundial de la Salud, oms, Reglamento Sanitario Internacional, Documento wHA 58.3, Ginebra, 2005.

Organización Mundial de la Salud, oms, Gro Harlem Brundtland, Speech to the Fifty-first World Health Assembly, A51/DIV/6, 13 de mayo de 1998.

Organización Mundial de la Salud, oms, Constitución de la Organización Mundial de la Salud, Washington D.C., 22 de junio de 1946.

Organización Mundial de la Salud/Organización Panamericana de la Salud oms/ops, Agreement between the World Health Organization and the Pan American Sanitary Organization, A2/VR/1O, Washington D. C., 24 de mayo de 1949.

Organización Panamericana de la Salud, ops, "Cluster de salud Colombia: sistema de información”, 2020, https://www.paho. org/es/cluster-salud-colombia-sistema-informacion (consulta del 20 de noviembre de 2020).

Organización Panamericana de la Salud, ops, "Informes de situación de la COVID-19”, 2020, https://www.paho.org/es/in formes-situacion-covid-19 (consulta del 20 de noviembre de 2020).

Organización Panamericana de la Salud, ops, "COVID-19 EMT Response”, Covid-19 PAHO/WHO Response Report núm. 33, 9 de noviembre de 2020.

Organización Panamericana de la Salud, ops, "Respuesta de la Organización Panamericana de la Salud a la COVID-19 en la región de las Américas, del 17 de enero al 31 de mayo del 2020", 10 de julio de 2020, https://iris.paho.org/handle/10665.2/52 454 (consulta del 20 de noviembre de 2020).

Organización Panamericana de la Salud, ops, "Presupuesto por Programas de la OPS 2020-2021”, Informe CD57-OD358, 5 de septiembre de 2019.

Organización Panamericana de la Salud, ops, "COVID-19 Information System for the Region of the Americas”, 2020, https:/ / paho-covid19-response-who.hub.arcgis.com/ (consulta del 20 de noviembre de 2020). 
Orton, J. Douglas y Karl E. Weick, "Loosely Coupled Systems: A Reconceptualization", The Academy of Management Review, vol. 15, núm. 2, 1990, pp. 203-223.

Oye, Kenneth A., Cooperation under Anarchy, Princeton, University Press, 1986.

Oye, Kenneth A., "Explaining Cooperation under Anarchy: Hypotheses and Strategies", World Politics, vol. 38, núm. 1, 1985, pp. 1-24.

Raustiala, Kal y David G. Victor, "The Regime Complex for Plant Genetic Resource's”, International Organization, vol. 58, núm. 2, 2004, pp. 277-309.

Rodrigues, Gilberto M. A. y Alberto Kleiman, "Covid-19: ¿una nueva oportunidad para el multilateralismo?”, Foreign Affairs Latinoamerica, vol. 20, núm. 3, 2020, pp. 36-46.

Rosenau, James N., y Ernst-Otto Czempiel (eds.), Governance without Government: Order and Change in World Politics, Cambridge, University Press, 1992.

Rosenthal, Uriel, Arjen Boin y Louise K. Comfort (eds.), Managing Crisis: Threats, Dilemmas, Opportunities, Springfield, Charles C. Thomas Publishers, 2001.

Rosenthal, Uriel, Paul 't Hart y Michael T. Charles, "The World of Crises and Crisis Management”, en Uriel Rosenthal, Paul 't Hart y Michael T. Charles (eds.), Coping with Crises: The Management of Disasters, Riots and Terrorism, Springfield, Charles C. Thomas Publishers, 1989, pp. 3-36.

SAndler, Todd, Global Collective Action, Cambridge, University Press, 2004.

Stephenson, Max Jr., "Making Humanitarian Relief Networks More Effective: Operational Coordination, Trust and Sense Making”, Disasters, vol. 29, núm. 4, 2005, pp. 337-350.

Trondal, Jarle, "The Autonomy of Bureaucratic Organizations: An Organization Theory Argument", Journal of International Organizations, vol. 5, núm. 2, 2014, pp. 54-68.

WeIK, Karl E., "Educational Organizations as Loosely Coupled Systems", Administrative Science Quarterly, vol. 21, núm. 1, 1976, pp. 1-19. 
FI LXI-2 LA OPS y la OMS FRente a LA PANDEMia de Covid-19 EN AL 331

Weiss, Thomas G., Global governance. In What? Why? Whither?, Cambridge, Polity Press, 2013.

Young, Oran R., International Cooperation, Ithaca, Cornell University Press, 1989. 
